ORGANOIDS

\section{Modelling PDAC-niche adaption}

Cell Stem Cell 22, 454-467 (2018).

Pancreatic ductal adenocarcinoma (PDAC) is highly chemoresistant due to extensive mutational heterogeneity and a dense stromal environment. As some patients benefit more from current therapies than others, there is a need to characterise and stratify individual tumours to predict clinical responses. Sato and colleagues used patient-derived PDAC organoids to recapitulate cancer features, modulate them and study tumour dependence on the niche.

The authors established a library of 39 PDAC-derived organoid lines, assessed driver gene mutations and, using xenografts, confirmed resemblance to parental tumours. They found that different driver gene mutations initiated the requirement of different niche factors to sustain organoid growth.

Three functional PDAC organoid subtypes were identified based on their reliance on Wnt ligands from the niche. Cancerassociated fibroblasts could act as ligand sources for PDAC organoids that critically depended on them, as demonstrated by co-transplantation experiments. A subtype that cell-autonomously expressed epithelial Wnts was niche-independent and associated with a more aggressive phenotype. PDAC organoids could acquire Wnt niche independency by downregulating GATA6, which initiated epithelial Wnt expression. Using engineered organoids with four PDAC driver mutations alone or in combination, acquisition of mutation-specific niche independence could be verified.
This study elegantly demonstrates the value of organoids as functional platforms to study adaptive responses and progression of pancreatic tumours with different molecular profiles.

https://doi.org/10.1038/s41556-018-0115-2

\section{ORGANOIDS \\ Organoids test drug response Science 359, 920-926 (2018).}

Co-clinical trials evaluate drug responses from patients enrolled in a parallel clinical trial using lab-based approaches, which traditionally rely on mouse models or xenografts. Valeri and colleagues created an organoid biobank from 71 patients with metastatic cholangiocarcinoma, colorectal or gastroesophageal cancer that were enrolled in phase I/II clinical trials, to evaluate molecular profiles and drug responses.

Histological and transcriptomic assessment confirmed similarities between organoids and original tumours, whereas intratumour heterogeneity was evident between primary cancer and metastases. Drug screening confirmed cancer organoid responsiveness if known mutations in signalling pathways were targeted with their respective inhibitors. The authors found a high clinical predictive value when comparing organoid data to 21 cases of patient responses, such as sensitivities to paclitaxel, cetuximab and others. To assess the response to regorafenib, organoids from responsive or resistant patients were xenografted into

\title{
ORGANOIDS
}

\section{A biobank for bladder cancer}

Diverse clinical outcomes in bladder cancers result from varying molecular profiles. Shen and colleagues generated a biobank of patient-derived bladder cancer organoids to study tumour heterogeneity, evolution and drug response.

Organoid lines were established from 16 patients with lesions ranging from low-grade, non-muscle-invasive disease to high-grade, muscle-invasive cancer, during different time points. Using orthotopic xenografts in immunodeficient mice, the authors could confirm similar histological and mutational profiles between organoids and primary tumours. Deep sequencing indicated that the tumour genotype was largely maintained in culture, although many organoids underwent clonal evolution during serial passaging. Interestingly, a tendency to transition to a basal phenotype was evident in culture, which was reversible in xenografts. Effects of 50 compounds with established relevance for bladder cancer were evaluated, with responses showing partial correlations with mutational profiles. Recurrent cancer organoids demonstrated resistance to a wider range of drugs. Based on the mutational profiles of these lines it was possible to predict drug responses, some of which were recapitulated in subsequent xenograft experiments.

Overall the authors confirm the value and effectiveness of 3D bladder cancer organoids as preclinical models and for drug screening.

https://doi.org/10.1038/s41556-018-0114-3

mice, and showed reduced vasculature and tumour volume only in sensitive patientxenografts. Grafts from metastasis-derived organoids were used to assess the initial response to regorafenib and progression after the emergence of resistance, indicative of tumour evolution. Clinical and preclinical responses to a treatment for chemorefractory colorectal cancer, confirming the ability of organoids to recapitulate intra-patient heterogeneity.

Overall, the authors reported $100 \%$ sensitivity, $93 \%$ specificity and a positive predictive value of $88 \%$ for patient drug responses, highlighting the efficiency of organoids in providing molecular insights and accurate predictions for drug responses.

https://doi.org/10.1038/s41556-018-0116-1

\section{ORGANOIDS}

\section{Gene corrections in sight Stem Cell Rep. 10, 1267-1281 (2018).}

Retinitis pigmentosa is an inherited retinopathy caused by mutations in the RPGR gene, which leads to blindness. To study disease mechanisms, Jin and colleagues reprogrammed urinary cells from three patients into inducible pluripotent stem cells (iPSCs) and generated 3D self-forming retinal organoids.

After long-term differentiation, the authors obtained rod photoreceptor cells with outer segments and electro-physiological properties. Patient organoids showed abnormal photoreceptor layer morphology, dislocation of opsins, shorter outer segments and impaired rhodopsin transport. Gene expression associated with photoreceptor maturation and phototransduction was reduced, indicating defects in photoreceptor function. RPGR mutation were then prepared in one iPSC line using CRISPR-Cas9 gene editing, which restored cell morphology, rod and cone numbers, expression patterns of photoreceptor-related genes and GFAP proteins, electro-physiological properties, and decreased apoptosis markers in the inner organoid layer. As RPGR contributes to ciliogenesis, cilia lengths in patient urinary cells, iPSCs, iPSC-derived retinal pigment epithelium and 3D retinae were also assessed and were shorter compared to controls. RPGR-corrected patient iPSCs and retinal pigment epithelium, however, exhibited normal cilia lengths. Overall, the patientderived organoids accurately recapitulated disease phenotypes, which could be rescued using gene correction. 